\title{
Endovascular repair for retrograde type $A$ intramural hematoma with focal intimal disruption in descending aorta
}

\author{
Jiehua Li ${ }^{1,2 \#}$, Xiaolong Zhang ${ }^{1,2 \#}$, Yuan Peng ${ }^{1,2}$, Lunchang Wang ${ }^{1,2}$, Tun Wang ${ }^{1,2}$, Xin $\mathrm{Li}^{1,2}$, Hao He ${ }^{1,2}$, \\ Quanming Li ${ }^{1,2}$, Chang Shu ${ }^{1,2,3} \wedge$ \\ ${ }^{1}$ Department of Vascular Surgery, the Second Xiangya Hospital, Central South University, Changsha, China; ${ }^{2}$ Vascular Disease Institute, Central \\ South University, Changsha, China; ${ }^{3}$ Center of Vascular Surgery, Fuwai Hospital, National Center for Cardiovascular Disease, Chinese Academy of \\ Medical Sciences and Peking Union Medical College, Beijing, China \\ Contributions: (I) Conception and design: J Li, C Shu; (II) Administrative support: J Li, C Shu; (III) Provision of study materials or patients: H He, Q \\ Li, C Shu; (IV) Collection and assembly of data: X Zhang, Y Peng, L Wang, T Wang, X Li; (V) Data analysis and interpretation: J Li, X Zhang, Y \\ Peng, L Wang, T Wang, X Li; (VI) Manuscript writing: All authors; (VII) Final approval of manuscript: All authors. \\ \#These authors contributed equally to this work. \\ Correspondence to: Chang Shu. Department of Vascular Surgery, the Second Xiangya Hospital, Central South University, 139 Middle Renmin Road, \\ Changsha, Hunan 410011, China. Email: shuchang@csu.edu.cn.
}

Background: This study aims to report the experience of a single center using thoracic endovascular aortic repair (TEVAR) to treat retrograde type A intramural hematoma (IMH) with focal intimal disruption (FID) in descending aorta.

Methods: A total of 24 consecutive patients with retrograde type A IMH and complicated with FID in descending aorta underwent TEVAR in our center from 2015 to 2020. Their clinical data, imaging manifestation and follow-up results were retrospectively reviewed and analyzed.

Results: The median age of patients was 57.9 years (range, $42-80$ years) and 18 were men (75\%). As the preoperative CT angiography showed, the 24 patients developed IMH complicated with different kinds of FIDs in descending aorta [5 had intramural blood pool (IBP), 15 had ulcer-like projection (ULP), 2 had penetrating atherosclerotic ulcer (PAU), and 5 had localized dissection]. Successful deployment of aortic stent graft was achieved in all patients. There was no endoleak, stent graft migration, spinal cord ischemia, stroke, or 30-day mortality observed after TEVAR. The median duration of follow-up was 30.0 months (range, 3-60 months). As the last follow-up CT angiography showed, most of the patients ( 23 in $24,96 \%$ ) had favorable aortic remodeling. The maximum hematoma thicknesses and maximum diameters of both ascending and descending aorta were significantly decreased. During follow-up, 1 patient developed retrograde type A aortic dissection (RAAD) and underwent open surgery 3 months after TEVAR. 1 patient died of lung cancer 2 years later. There was no aorta-related death observed.

Conclusions: TEVAR provides a safe and effective treatment strategy for selected patients with retrograde type A IMH, and FID developed in descending aorta could be the possible treatment target. However, RAAD remains one of the most serious postoperative complications of concern.

Keywords: Thoracic endovascular aortic repair (TEVAR); retrograde type A intramural hematoma (retrograde type A IMH); focal intimal disruption (FID); aortic stent graft

Submitted Mar 31, 2021. Accepted for publication Jun 04, 2021.

doi: $10.21037 /$ jtd-21-574

View this article at: https://dx.doi.org/10.21037/jtd-21-574

\footnotetext{
^ ORCID: 0000-0002-5096-4655.
} 


\section{Introduction}

Aortic intramural hematoma (IMH) is an emergency and life-threatening medical condition belonging to the spectrum of acute aortic syndrome (1). Unlike the classic aortic dissection, IMH is characterized by the presence of a hematoma within the aortic wall and manifests as crescent or circular aortic wall thickening. Although IMH doesn't present as overt false lumen, focal intimal disruption (FID) could be detected in $70 \%$ of IMH with high resolution computed tomography (2). The FIDs include ulcer-like projection (ULP), penetrating atherosclerotic ulcer (PAU), intramural blood pool (IBP) and localized dissection, which are regarded as risk factors for the deterioration of IMH (3-5).

The optimal treatment for IMH remains controversial, especially for those involving ascending aorta. The evolution of IMH could be quite elusive, as it could be completely absorbed, progress to aortic dissection or even cause aortic rupture. Retrograde type A IMH is a special subtype of IMH that develops in a retrograde way with primary intimal disruption located in descending aorta. Open surgery was thought to be the most effective treatment for type A IMH, though initial medical treatment showed good outcomes in selected patients $(6,7)$. However, open surgery is usually associated with high mortality and morbidity, and maybe not suitable for patients with poor status. Thoracic endovascular aortic repair (TEVAR) is a less invasive alternative and its value for descending thoracic aortic diseases has been well confirmed (8). And recently several studies have reported the application of TEVAR in retrograde type A IMH or aortic dissection or IMH to exclude the intimal tear in descending aorta (9-11). TEVAR could be a possible treatment strategy for patients with retrograde type A IMH, but its safety and efficacy are not fully elucidated.

This study is to describe the experience of a single center using TEVAR to treat retrograde type A IMH with FID in descending aorta in selected patients. We present the following article in accordance with the STROBE reporting checklist (available at https://dx.doi.org/10.21037/jtd-21-574).

\section{Methods}

\section{Study design and patient enrollment}

This retrospective study was performed with approval of the Institutional Ethics Board of the Second Xiangya Hospital of Central South University (NO. 2020S069) and informed consent was obtained from every patient. The study was conducted in accordance with the Declaration of Helsinki (as revised in 2013). From January $1^{\text {st }} 2015$ to December $31^{\text {st }} 2020$, a total of 24 consecutive patients with retrograde type A IMH and complicated with FID in descending aorta underwent TEVAR in our center. Their medical records were retrospectively reviewed and analyzed. Patients who met the following criteria were considered to be candidates for TEVAR: (I) there was no intimal disruption in ascending aorta and proximal aortic arch; (II) the maximum hematoma thickness of ascending aorta was no more than $15 \mathrm{~mm}$, and maximum diameter of ascending aorta was no more than $55 \mathrm{~mm}$; (III) there was no signs of cardiac tamponade, severe aortic regurgitation, or involvement of the coronary artery by hematoma. Before the operation, a multidisciplinary board was consulted to determine the operation strategy, which included vascular and cardiovascular surgeons, an anesthesiologist and a radiologist. Patient enrollment of the study was presented in Figure 1.

IMH was diagnosed as the presence of a circular or crescent-shaped thickening of at least $5 \mathrm{~mm}$ of the aortic wall with the absence of visible intimal flap in CT angiography. The FID types including ULP, PAU, IBP and localized dissection were defined according to previous publications (3-5). All the 4 types of FIDs could be well detected by CT angiography: ULP protrudes from the lumen to thrombosed media, PAU is the ulceration through an atherosclerotic plaque, IBP has no obvious communication with the lumen, while localized dissection has the manifestation of "true and false lumen". The measurement of maximum hematoma thickness and maximum diameter of both ascending and descending aorta were performed by three personnel and the average value was chosen in the study.

\section{Operative details and device selection}

All patients received a CT angiography at admission, and medical treatment with antihypertensive drugs and betablockers were administrated immediately. Those with stable conditions would take a repeated CT angiography about one week later. TEVAR is indicated if the patient presented recurrent chest pain, uncontrollable hypertension, significant progression of IMH or FID. Among the 24 patients, 21 underwent standard TEVAR as previously reported $(9,12)$, while 1 underwent TEVAR with chimney technique and 2 underwent TEVAR with physician modified fenestration to preserve left subclavian artery as we previously reported (13). As the treatment goal is to 


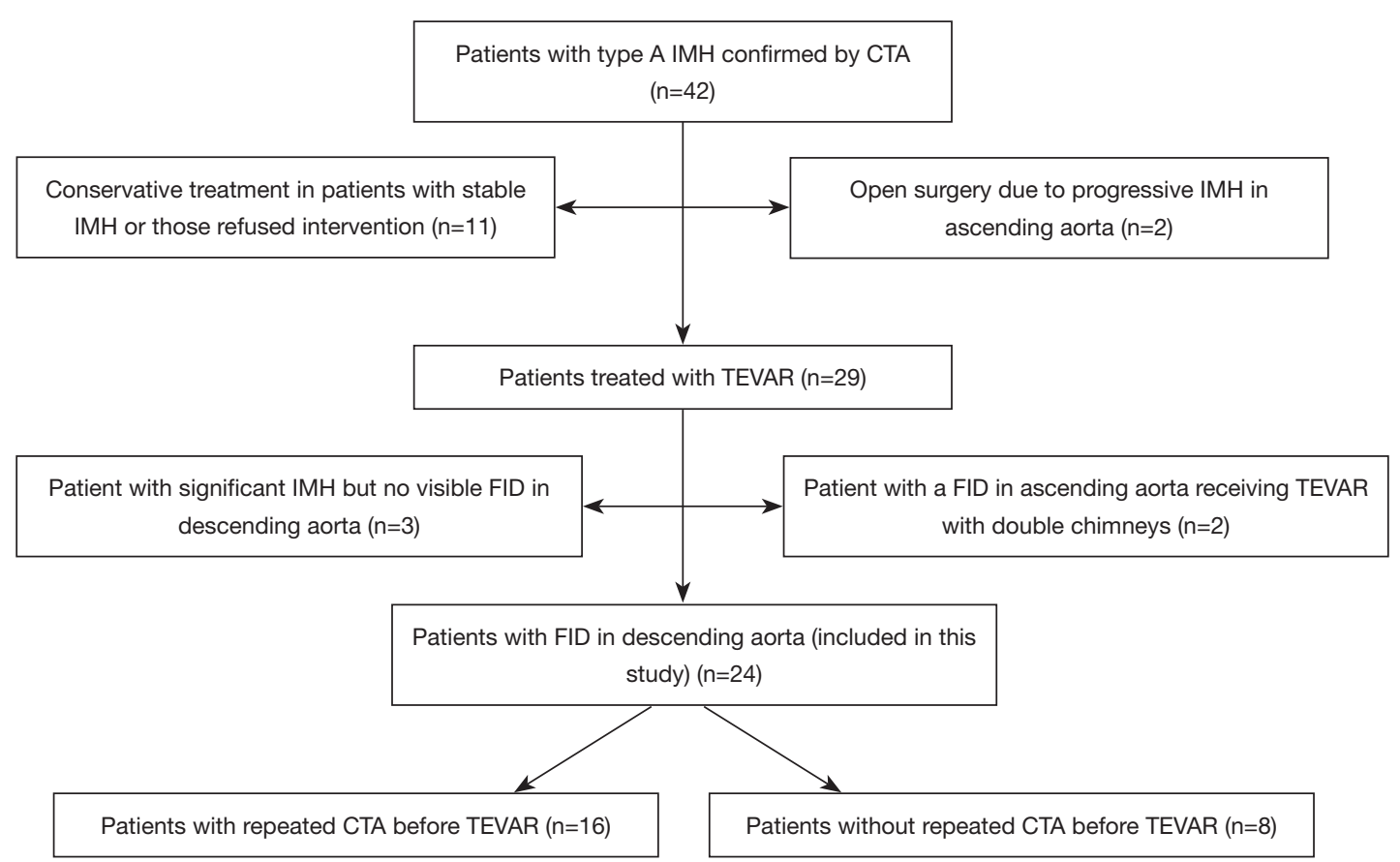

Figure 1 Flow chart for patient enrollment.

cover FID in descending aorta, there should be at least 2 $\mathrm{cm}$ of proximal and distal sealing. The procedures were performed in a hybrid unit with fluoroscopic guidance, and general anesthesia with tracheal intubation was applied in all patients.

The selected diameter size of the stent graft was $10-15 \%$ greater than the diameter of the aorta at the proximal sealing zone measured in aortic angiography. Three types of commercially available stent grafts were implanted in the 24 patients. The Medtronic Valiant thoracic endovascular stent graft (Medtronic, Inc., Minneapolis, MN, USA) was used in 6 patients. The MicroPort Hercules stent graft (MicroPort Medical Shanghai Co, Ltd., Shanghai, China) was used in 2 patients. The Lifetech Ankura stent graft (Lifetech Scientific Co, Ltd., Shenzhen, China) was used in the rest 16 patients.

\section{Follow-up}

All of the patients were scheduled with follow-up physical examination and CT angiography 2 weeks, 3 months, 6 months, and 12 months after TEVAR, and annually thereafter to evaluate stent-graft position, endoleaks, and changes of aortic pathologies. Survival was assessed by outpatient clinic visit or telephone interview.

\section{Statistical analysis}

Descriptive statistics were used to describe patient data and outcomes in this cohort. The values were expressed as mean \pm standard deviation or as medians (ranges). Normality was assessed using the Shapiro-Wilk test. The maximal diameters of aorta and maximum hematoma thickness before and after TEVAR were compared with Student paired $t$-test. $\mathrm{P}<0.05$ was considered statistically significant. Statistical analysis was conducted using GraphPad 7.0 (GraphPad Software, Inc., San Diego, CA, USA).

\section{Results}

\section{Baseline characteristics and preoperative imaging manifestation}

The median age of the 24 patients was 57.9 years (range, $42-80$ years) and 18 were men $(75 \%)$. Their baseline characteristics were presented in Table 1 . There were 6 patients complicated with pericardial effusion and 13 patients with pleural effusion, but drainage of the effusions was not required.

The preoperative imaging manifestations were summarized in Table 2. The 24 patients developed different types of FIDs (5 had IBP, 15 had ULP, 2 had PAU, and 
Table 1 Baseline characteristics of the 24 patients

\begin{tabular}{lcc}
\hline Variables & Number or mean & Range or \% \\
\hline Age, y & $57.9 \pm 7.1$ & $42-80$ \\
Male & 18 & $75 \%$ \\
Comorbidities & 23 & \\
Hypertension & 3 & $96 \%$ \\
Coronary heart disease & 2 & $13 \%$ \\
Diabetes & 2 & $8 \%$ \\
Renal dysfunction & 2 & $8 \%$ \\
COPD & 1 & $8 \%$ \\
History of stroke & 12 & $4 \%$ \\
Smoking & 13 & $50 \%$ \\
Pleural effusion & 6 & $54 \%$ \\
Pericardial effusion & $25 \%$ \\
\hline
\end{tabular}

COPD, chronic obstructive pulmonary diseases.
5 had localized dissection) in descending aorta. Among them, 1 had both ULP and IBP, and 2 had ULP that progressed to localized dissection. Twenty patients had crescent shape of hematoma, and 4 patients presented with circular shape. Sixteen patients had more than one preoperative CT angiography. Four patients had newly developed FIDs (1 with ULP, 1 with ULP and IBP, 2 with localized dissections) in late repeated CT angiography, while presented without FID in the initial CT angiography on admission. Nine patients showed progressed FIDs, among whom 4 had significant enlarged ULP in the second CT angiography, and the ULP in 2 progressed to localized dissection after 7 days' medical treatment (Figure $2 A, B, C, D), 1$ had enlarged PAU, and 2 developed progressive IBP (Figure 3A,B,C),

\section{Perioperative results}

Successful deployment of aortic stent graft was achieved in

Table 2 Preoperative imaging manifestations

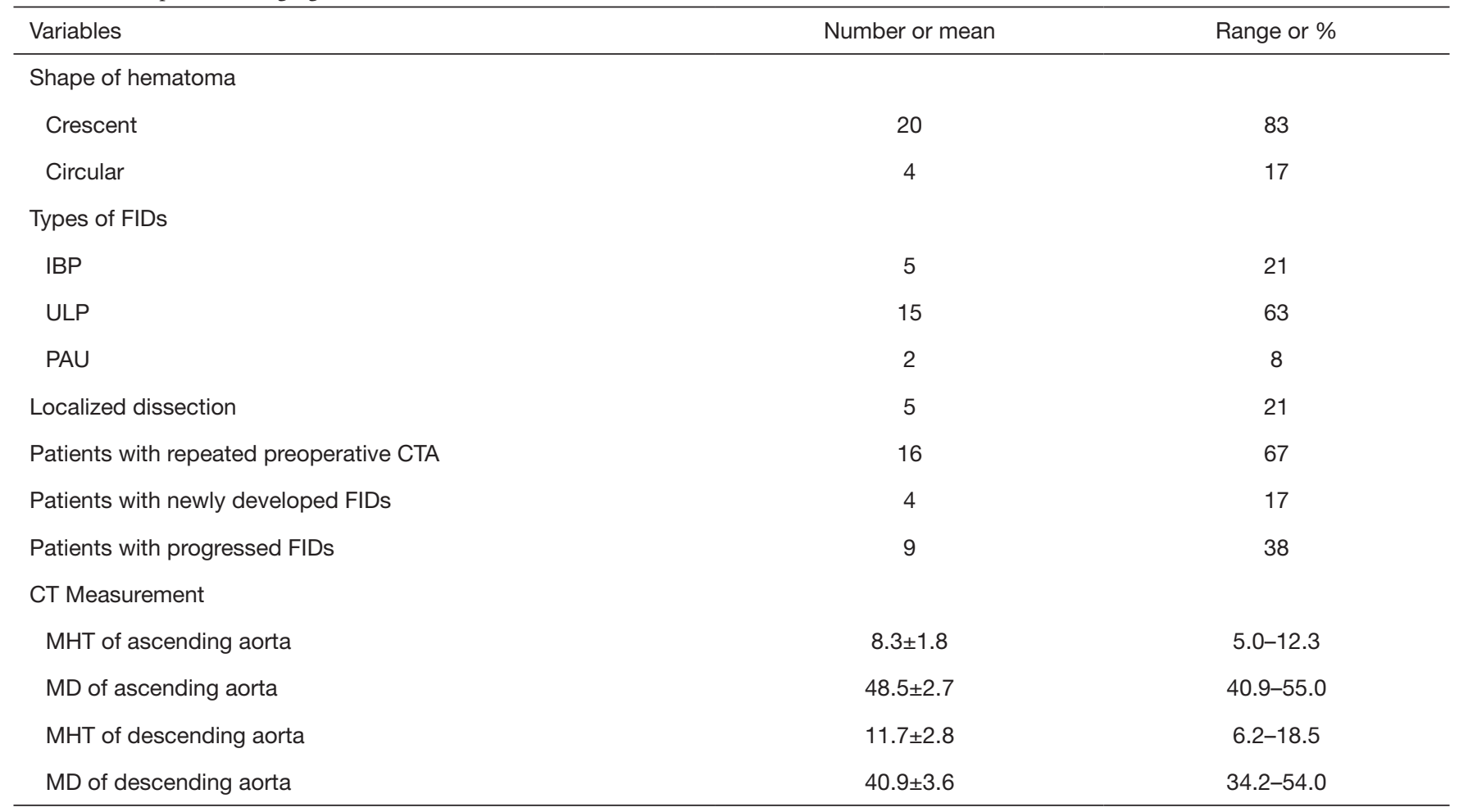

FID, focal intimal disruption; IBP, intramural blood pool; ULP, ulcer-like projection; PAU, penetrating atherosclerotic ulcer; MHT, maximum hematoma thickness; MD, maximum diameter. 

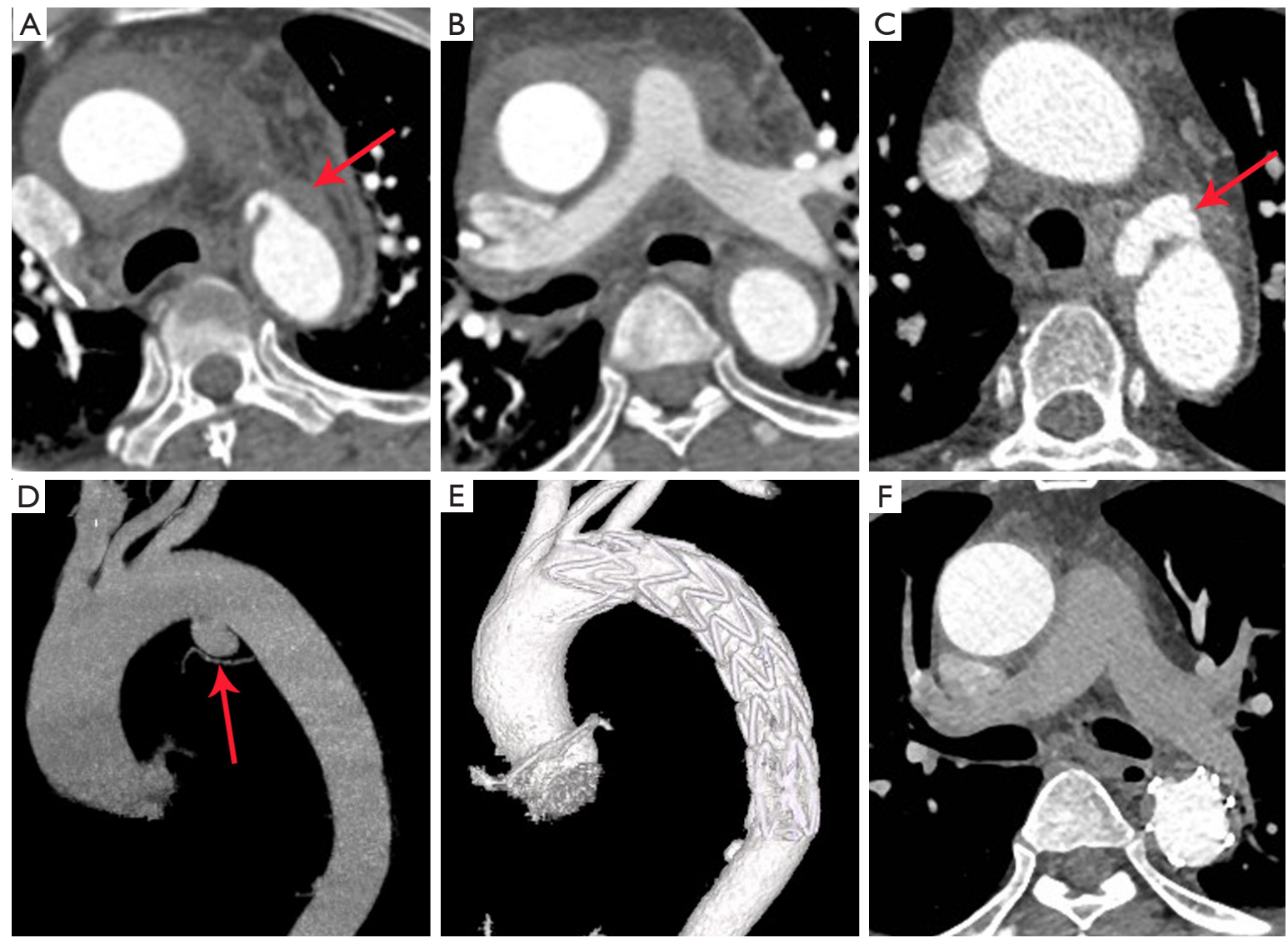

Figure 2 TEVAR for retrograde type A IMH with a progressive ULP in descending aorta. CT angiography on admission showed retrograde type A IMH with a ULP in descending aorta (red arrow showed) (A,B). After 1 week's medical treatment, the CT angiography showed the ULP had progressed to a localized dissection (red arrow showed) (C,D). Then the patient underwent TEVAR (E), and CT angiography demonstrated good aortic remodeling 3 months later (F). TEVAR, thoracic endovascular aortic repair; IMH, intramural hematoma; ULP, ulcer-like projection.

all patients. The perioperative and follow-up results were shown in Table 3. Among the 24 patients, 12 underwent TEVAR in acute phase (1-14 days), 7 underwent TEVAR in sub-acute phase (15-30 days), and the remaining 5 patients underwent TEVAR in chronic phase ( $>30$ days after symptom onset). One patient underwent TEVAR as long as 11 months since the first admission, as he had recurrent chest pain and CT angiography revealed a newly developed ULP. The mean operative time was $78.5 \pm 14.7 \mathrm{~min}$ (range, 55-120 $\mathrm{min}$ ). One patient entered ICU after TEVAR, and the ICU stay was 2 days. Chest radiography and echocardiography showed that the pericardial and pleural effusion decreased after TEVAR and were mostly re-absorbed 7-30 days later.

There was no aortic rupture, neurological defect, access site complication, spinal cord ischemia, endoleak or stent graft migration observed after TEVAR. And there was no 30-day mortality.

\section{Follow-up outcome and aortic remodeling}

There were 23 patients kept in follow-up after discharge. One patient was lost to follow-up 3 months after TEVAR. The mean duration of follow-up was $30.0 \pm 16.9$ months (range, 3-60 months ). One patient developed retrograde type A aortic dissection (RAAD) and underwent open surgery 3 months after TEVAR. 1 patient died 2 years after TEVAR due to progression of lung cancer. There was no other adverse events or aorta-related death observed during follow-up.

All the patients had satisfactory aortic remodeling after TEVAR (as Figures 1,2 showed), except the one who developed RAAD. The last follow-up CT angiography 

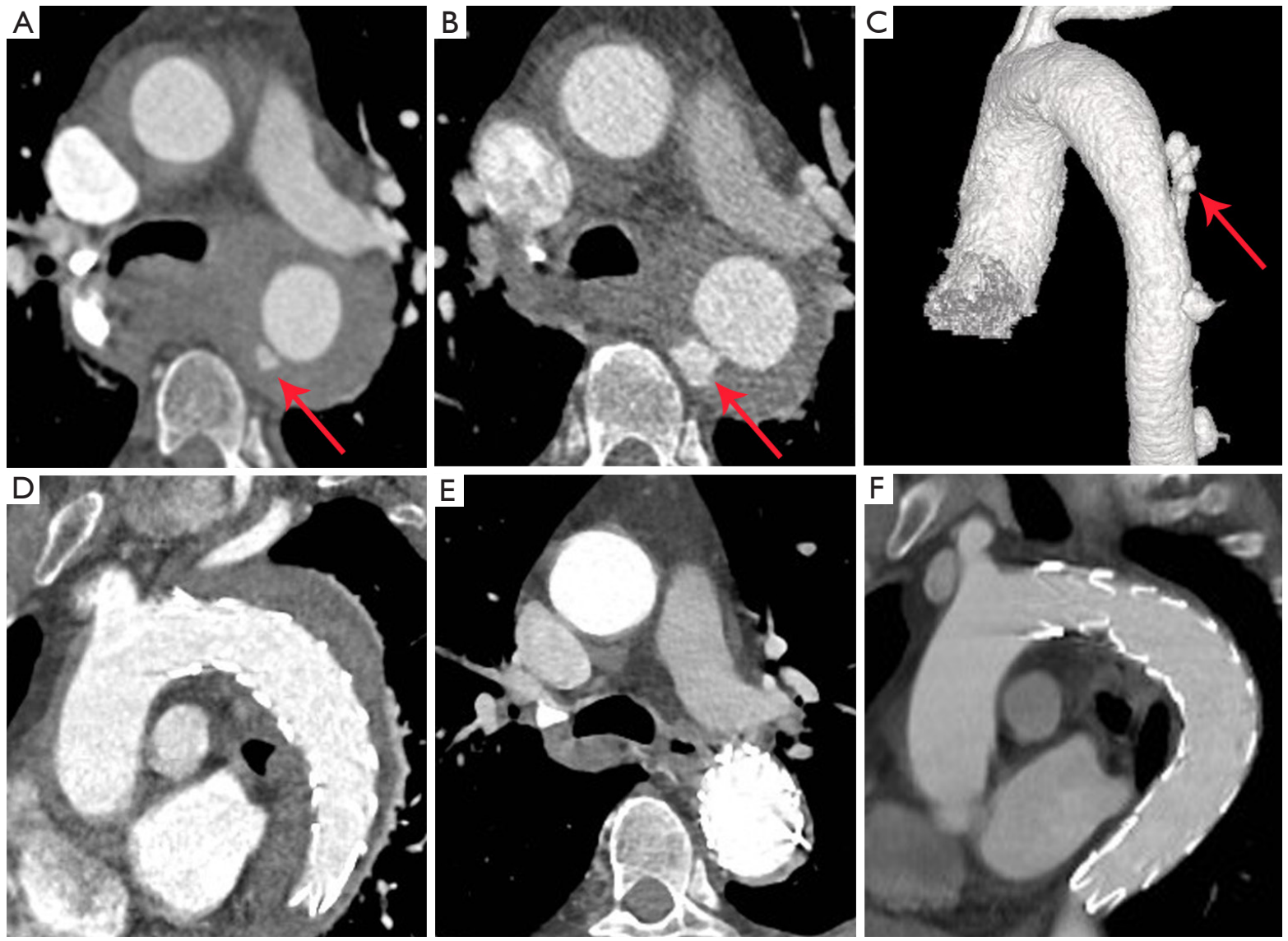

Figure 3 TEVAR for retrograde type A IMH with progressive IBPs in descending aorta. CT angiography on admission showed retrograde type A IMH with an IBP in descending aorta (red arrow showed) (A). After 1 week's medical treatment, CT angiography showed the IBP had significantly progressed (red arrow showed) (B,C). Then TEVAR was performed (D). CT angiography demonstrated good aortic remodeling 3 months later (E,F). TEVAR, thoracic endovascular aortic repair; IMH, intramural hematoma; IBP, intramural blood pool.

demonstrated the maximum hematoma thicknesses of both ascending and descending aorta were significantly decreased (from $8.3 \pm 1.8$ to $3.0 \pm 1.8 \mathrm{~mm}, \mathrm{P}<0.05$, and from $11.7 \pm 2.8$ to $3.7 \pm 2.0 \mathrm{~mm}, \mathrm{P}<0.05$ ). And the maximum diameters of both ascending and descending aorta were also significantly decreased (from $47.5 \pm 2.7$ to $42.7 \pm 3.6 \mathrm{~mm}, \mathrm{P}<0.05$, and from $40.9 \pm 3.6$ to $34.6 \pm 3.0 \mathrm{~mm}, \mathrm{P}<0.05$ ) (Figure $4 A, B, C, D$ ). Complete resolution of IMH (maximum hematoma thicknesses $<3 \mathrm{~mm}$ ) in the ascending aorta was found in 18 patients $(75 \%)$, and complete resolution of IMH in the descending aorta occurred in 13 patients $(54 \%)$.

\section{Discussion}

The present study tried to verify the effect and safety of TEVAR in selected patients with retrograde type A IMH and FID in descending aorta. During a period of 6 years, a series of 24 consecutive patients were treated with TEVAR. The early and mid-term outcomes showed low incidence of adverse complications and favorable aortic remodeling after operation. IMH in both ascending aorta and descending aorta were significantly decreased. The ascending aorta seemed to have a higher rate of complete resolution of $\mathrm{IMH}$, possibly due to the fact that IMH in ascending aorta was generally milder than descending aorta.

The management of IMH could be challenging, mostly because of its variable natural history and highly unpredictable behavior. FID has prognostic value for IMH, and may provide guidance for clinical decision-making (5). The patients in our series presented different types of FIDs (IBP in 5, ULP in 15, PAU in 2, LD in 5). Twenty patients had FIDs in the CT angiography at admission, while 4 
Table 3 Perioperative and follow-up results

\begin{tabular}{lcc}
\hline Variables & Number or mean & Range or $\%$ \\
\hline Operation time, minutes & $78.5 \pm 14.7$ & $55-120$ \\
Hospital stay, days & $20.4 \pm 3.5$ & $13-28$ \\
Patients lost follow-up & 1 & 4 \\
Follow-up time, months & $30.0 \pm 16.9$ & $3-60$ \\
Interval from symptom onset & & \\
to TEVAR & 12 & 50 \\
1-14 d & 7 & 29 \\
15-30 d & 5 & 21 \\
$>30$ d & 0 & 0 \\
30-day mortality & 1 & 4 \\
Type A aortic dissection & 0 & 0 \\
Re-intervention & 0 & 0 \\
Aorta-related mortality & 1 & 4 \\
All-cause mortality & &
\end{tabular}

TEVAR, thoracic endovascular aortic repair.

patients showed late developed FIDs in the secondary CT angiography. And 9 patients showed progressive changes of FIDs (4 with enlarged ULP, 1 with enlarged PAU, 2 with progressive IBP, and 2 with ULP progressed to localized dissection). The FIDs are also the targets for endovascular repair. Covering of FIDs could potentially block blood flow into the aortic wall and help to get better aortic remodeling.

Retrograde type A IMH is a special subtype of IMH with the primary intimal disruption located in descending aorta. The involvement of ascending aorta poses high risk for early progression of the disease, such as type A aortic dissection, tamponade, hemothorax, and even aortic rupture $(14,15)$. Although traditional surgical repair is thought to be an effective therapy for type A IMH, it is reported to be associated with significant morbidity and mortality and is not suitable for patients with poor conditions $(16,17)$.

TEVAR provides a less invasive alternative to open surgery in aortic diseases, and it has been widely used in treating descending aortic lesions (8). And with the progress of technology, such as chimney, fenestrated or branched stent grafts, some arch lesions could also be treated with TEVAR (18-20). Previous studies have demonstrated that the application of regular TEVAR to cover the entry tear in descending aorta is a feasible strategy to treat retrograde type A aortic dissection $(9,10,21)$. A systematic review of endovascular management of retrograde type A IMH or aortic dissection summarized that overall in-hospital mortality was $1.8 \%$, and additional all-cause mortality during follow-up was $5.4 \%$, which indicated that for some certain patients TEVAR might be superior to open surgery (22).

Several groups reported the application of TEVAR in type A IMH, mostly in case reports and small case series (23-26). Grimm et al. reported a series of 8 patients with type A IMH that were successfully treated with regular TEVAR (26). And they raised a novel theory, that the atherosclerotic plaque rupture at the free lateral wall or at the concavity of proximal arch would make IMH tend to affect the entire thoracic aorta, thus could be the targets of TEVAR. Their outcomes were satisfactory, with the hematoma in ascending aorta completely reabsorbed in all the 8 patients. And very recently, Ryoi et al. reported their experience with TEVAR for a series of 18 cases of retrograde type A IMH, among whom 11 presented aortic dissection and 7 presented IMH in descending aorta (11). Another group, Li et al. reported TEVAR for 15 cases with type $\mathrm{B}$ aortic dissection associated with retrograde type A IMH (27). Both of the studies showed satisfactory midterm outcomes with high survival rate and low incidence of adverse events. In the current study, all the 24 patients had IMH with FID in descending aorta and they underwent TEVAR with a low rate of postoperative complications observed. Based on the literature and our experience, TEVAR could be a feasible and effective therapy for retrograde type A IMH with FID in descending aorta.

The optimal timing for TEVAR applied in retrograde type A IMH remains a controversial issue. Typically, urgent surgical repair is recommended for type A IMH or aortic dissection, but studies have demonstrated that initial medical management with timely interventions or the "Watch-and-wait strategy" could achieve good outcomes for retrograde type A IMH or aortic dissection (28-30). As retrograde type A IMH or aortic dissection showed more favorable prognosis than antegrade type A IMH or aortic dissection which had intimal tear in ascending aorta. And the studies of TEVAR in acute type B aortic dissection suggested that appropriate delay of intervention could lower the risk of complications of TEVAR in patients that were stable to wait (31). In the current study, the average time interval between the onset of symptoms and TEVAR was $17.5 \pm 8.2$ days (range, $7-40$ days, with the case of 330 days excluded). The authors advocated appropriate delay of TEVAR in acute retrograde type A IMH and the rationales are as follows: firstly, the aortic wall in acute phase may be 
A

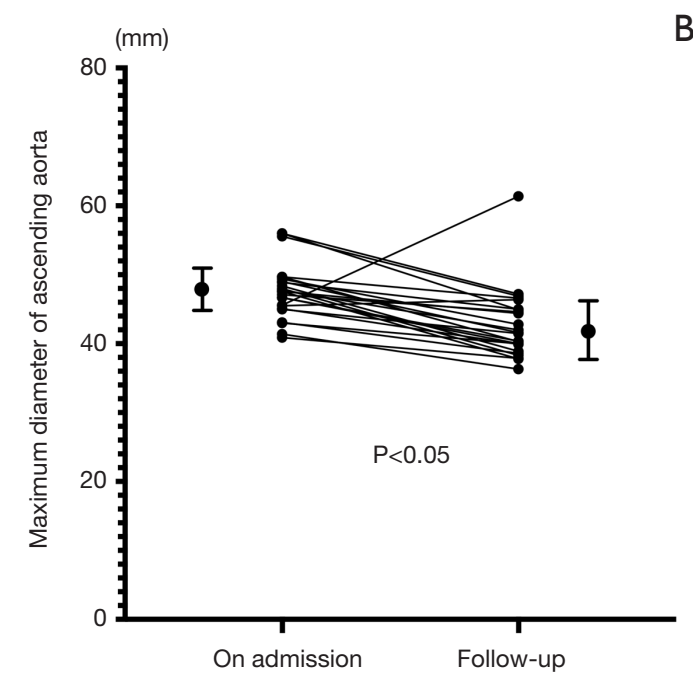

C

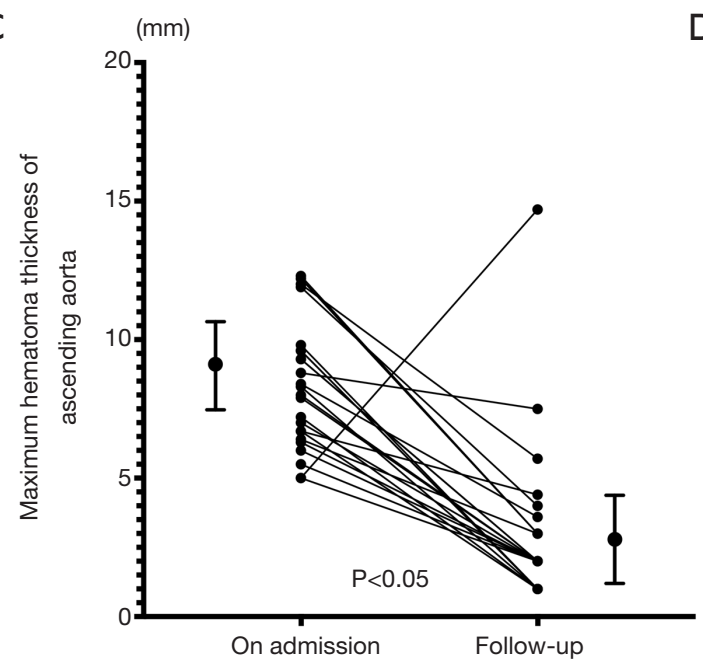

B
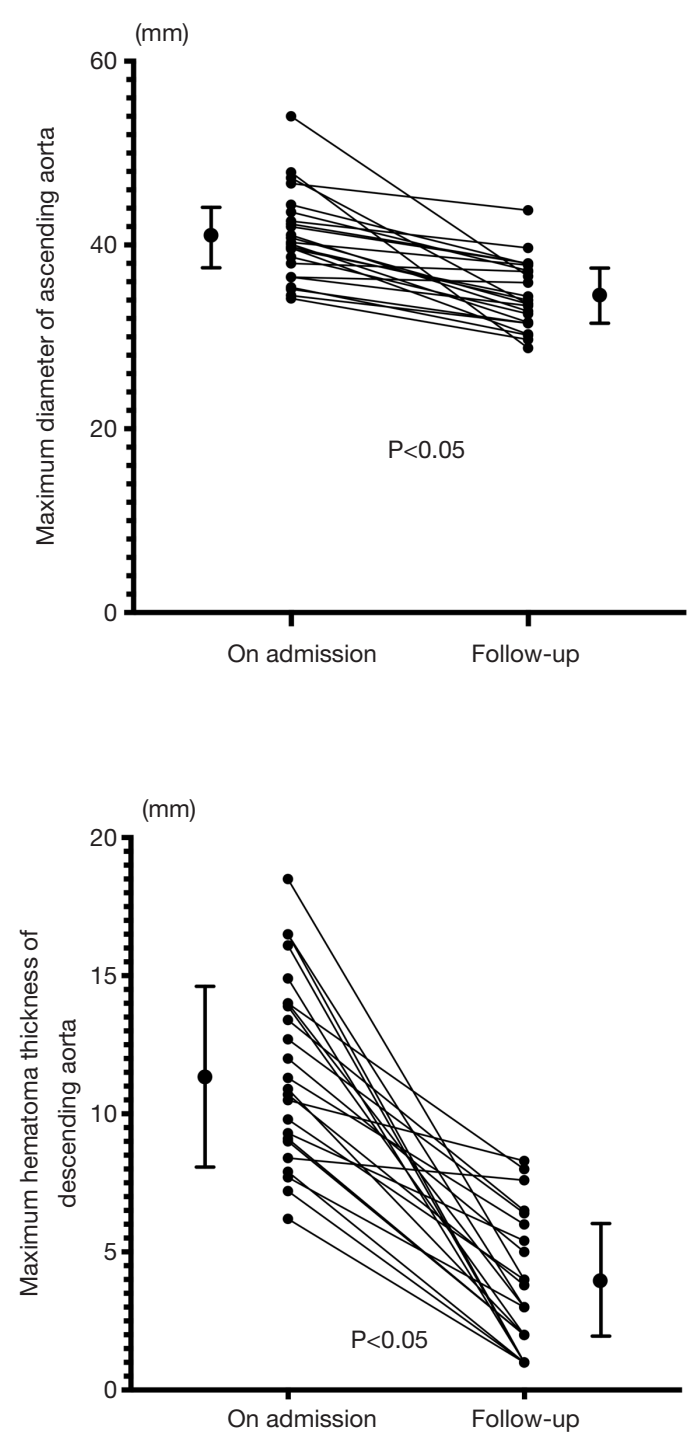

Figure 4 Aortic remodeling of the patients with retrograde type A IMH after TEVAR. The maximal diameters of ascending aorta (A) and descending aorta (B) in follow-up was significantly decreased compared with that on admission. Maximum hematoma thickness of ascending aorta (C) and descending aorta (D) in follow-up was significantly decreased compared with that on admission. Mean values \pm SD are shown in the dots with bars. IMH, intramural hematoma; TEVAR, thoracic endovascular aortic repair.

fragile to tolerate the deployed stent grafts; secondly, it's important to make sure there is no newly developed FID or significantly progressed hematoma in ascending aorta, which may otherwise indicate for open surgery.

It is the consensus that ideal proximal seal zone for TEAVR should be in a healthy aorta segment. As some investigators showed, landing on IMH could increase the risk of RAAD after TEVAR (32). For the patients with retrograde type A IMH undergoing TEVAR, development of a new RAAD is one of the most serious complications that should not be ignored. In the current series the rate of RAAD was $4 \%$, and another group also reported the occurrence of RAAD after TEVAR in retrograde type A IMH (24). There are several possible mechanisms. Firstly, as the stent grafts deployed on the aorta segment with $\mathrm{IMH}$, the intima could potentially be injured, especially when there is too much oversizing or a "bird-beak" formed. Secondly, the manipulation of wires and catheters during TEVAR could possibly cause damage to the intima of ascending aorta which already presented with IMH. 
Thirdly, there may be tiny and unrecognizable intimal tear in the IMH of ascending aorta, which could gradually enlarge and lead to the development of RAAD.

The study has several limitations. Firstly, it is a retrospective and observational study performed in a single center with a relatively small number of patients. Secondly, the study did not include the patients that underwent open surgery or received conservative treatment only. Thirdly, the duration of follow-up is limited, and longer follow-up and observation are needed to confirm the long-term safety.

\section{Conclusions}

The current study indicated that TEVAR could be a feasible therapy for retrograde type A IMH with focal intima disruption (FID) in descending aorta. In selected patients, TEVAR seemed to provide an effective and safe strategy with satisfactory early and midterm outcomes and favorable aortic remodeling. And the FID developed in descending aorta could be the possible treatment target for TEVAR. But RAAD remains one of the most serious postoperative complications of concern. And further studies are still warranted in future to testify the long-term durability and safety, as well as to optimize the intervention.

\section{Acknowledgments}

Funding: This work is supported by the National Natural Science Foundation of China (Grant No. 81800400) and the Natural Science Foundation of Hunan Province (Grant No. 2019JJ50851).

\section{Footnote}

Reporting Checklist: The authors have completed the STROBE reporting checklist. Available at https://dx.doi. org/10.21037/jtd-21-574

Data Sharing Statement: Available at https://dx.doi. org/10.21037/jtd-21-574

Peer Review File: Available at https://dx.doi.org/10.21037/ jtd-21-574

Conflicts of Interest: All authors have completed the ICMJE uniform disclosure form (available at https://dx.doi. org/10.21037/jtd-21-574). JL reports funding support from the National Natural Science Foundation of China (grant number 81800400) and the Natural Science Foundation of Hunan Province (grant number 2019JJ50851). The authors have no other conflicts of interest to declare.

Ethical Statement: The authors are accountable for all aspects of the work in ensuring that questions related to the accuracy or integrity of any part of the work are appropriately investigated and resolved. The study was approved by the Institutional Ethics Board of the Second Xiangya Hospital of Central South University (NO. 2020S069) and written informed consents were obtained from all patients. The study was conducted in accordance with the Declaration of Helsinki (as revised in 2013).

Open Access Statement: This is an Open Access article distributed in accordance with the Creative Commons Attribution-NonCommercial-NoDerivs 4.0 International License (CC BY-NC-ND 4.0), which permits the noncommercial replication and distribution of the article with the strict proviso that no changes or edits are made and the original work is properly cited (including links to both the formal publication through the relevant DOI and the license). See: https://creativecommons.org/licenses/by-nc-nd/4.0/.

\section{References}

1. Mussa FF, Horton JD, Moridzadeh R, et al. Acute Aortic Dissection and Intramural Hematoma: A Systematic Review. JAMA 2016;316:754-63.

2. Kitai T, Kaji S, Yamamuro A, et al. Detection of intimal defect by 64-row multidetector computed tomography in patients with acute aortic intramural hematoma. Circulation 2011;124:S174-8.

3. Tanaka A, Leake S, Estrera AL. Management strategies in acute type B aortic intramural hematoma. Curr Opin Cardiol 2017;32:687-91.

4. Maslow A, Atalay MK, Sodha N. Intramural Hematoma. J Cardiothorac Vasc Anesth 2018;32:1341-62.

5. Moral S, Cuéllar H, Avegliano G, et al. Clinical Implications of Focal Intimal Disruption in Patients With Type B Intramural Hematoma. J Am Coll Cardiol 2017;69:28-39.

6. Kaji S, Akasaka T, Horibata Y, et al. Long-term prognosis of patients with type a aortic intramural hematoma. Circulation 2002;106:I248-52.

7. Song JK, Yim JH, Ahn JM, et al. Outcomes of patients with acute type a aortic intramural hematoma. Circulation 2009;120:2046-52.

8. Cheng D, Martin J, Shennib H, et al. Endovascular aortic 
repair versus open surgical repair for descending thoracic aortic disease a systematic review and meta-analysis of comparative studies. J Am Coll Cardiol 2010;55:986-1001.

9. Shu C, Wang T, Li QM, et al. Thoracic endovascular aortic repair for retrograde type A aortic dissection with an entry tear in the descending aorta. J Vasc Interv Radiol 2012;23:453-60, 460.e1.

10. Higashigawa T, Kato N, Nakajima K, et al. Thoracic endovascular aortic repair for retrograde type A aortic dissection. J Vasc Surg 2019;69:1685-93.

11. Ryoi O, Lin CH, Chen JM, et al. Endovascular Repair for Retrograde Type A Intramural Haematoma with Intimal Tear in the Descending Thoracic Aorta. Eur J Vasc Endovasc Surg 2020;60:386-93.

12. Shu C, Fang K, Dardik A, et al. Pregnancy-associated type $B$ aortic dissection treated with thoracic endovascular aneurysm repair. Ann Thorac Surg 2014;97:582-7.

13. Shu C, Fan B, Luo M, et al. Endovascular treatment for aortic arch pathologies: chimney, on-the-table fenestration, and insitu fenestration techniques. J Thorac Dis 2020;12:1437-48.

14. Sandhu HK, Tanaka A, Charlton-Ouw KM, et al. Outcomes and management of type A intramural hematoma. Ann Cardiothorac Surg 2016;5:317-27.

15. Song JM, Kim HS, Song JK, et al. Usefulness of the initial noninvasive imaging study to predict the adverse outcomes in the medical treatment of acute type A aortic intramural hematoma. Circulation 2003;108 Suppl 1:II324-8.

16. Kamohara K, Furukawa K, Koga S, et al. Surgical strategy for retrograde type A aortic dissection based on long-term outcomes. Ann Thorac Surg 2015;99:1610-5.

17. Tamura K, Uchida N, Katayama A, et al. The frozen elephant trunk technique for retrograde acute type A aortic dissection. J Thorac Cardiovasc Surg 2014;148:561-5.

18. Crawford SA, Sanford RM, Forbes TL, et al. Clinical outcomes and material properties of in situ fenestration of endovascular stent grafts. J Vasc Surg 2016;64:244-50.

19. Tsilimparis N, Debus ES, von Kodolitsch Y, et al. Branched versus fenestrated endografts for endovascular repair of aortic arch lesions. J Vasc Surg 2016;64:592-9.

20. Wang T, Shu C, Li M, et al. Thoracic Endovascular Aortic Repair With Single/Double Chimney Technique for Aortic Arch Pathologies. J Endovasc Ther 2017;24:383-93.

21. Kato N, Shimono T, Hirano T, et al. Transluminal placement of endovascular stent-grafts for the treatment of type A aortic dissection with an entry tear in the descending thoracic aorta. J Vasc Surg 2001;34:1023-8.

22. Lyons O, Clough R, Patel A, et al. Endovascular management of Stanford type a dissection or intramural hematoma with a distal primary entry tear. J Endovasc Ther 2011;18:591-600.

23. Haenen FWN, Van Der Weijde E, Vos JA, et al. Retrograde Type A Intramural Hematoma Treated Endovascularly in Two Cases. Ann Vasc Surg 2019 Aug;59:312.e15-312.e18.

24. Chen YY, Yen HT, Wu CC, et al. Thoracic Endovascular Aortic Repair for Type A Intramural Hematoma and Retrograde Thrombosed Type A Aortic Dissection: A Single-Center Experience. Ann Vasc Surg 2020;65:224-31.

25. Monnin-Bares V, Thony F, Rodiere M, et al. Endovascular stent-graft management of aortic intramural hematomas. J Vasc Interv Radiol 2009;20:713-21.

26. Grimm M, Loewe C, Gottardi R, et al. Novel insights into the mechanisms and treatment of intramural hematoma affecting the entire thoracic aorta. Ann Thorac Surg 2008;86:453-6.

27. Li YL, Ye JC, Yancu H, et al. Thoracic Endovascular Aortic Repair for Type B Aortic Dissection Associated with Retrograde Type A Intramural Hematoma. J Vasc Interv Radiol 2020;31:1334-41.

28. Kim JB, Choo SJ, Kim WK, et al. Outcomes of acute retrograde type A aortic dissection with an entry tear in descending aorta. Circulation 2014;130:S39-44.

29. Kitamura T, Torii S, Miyamoto T, et al. Watch-andwait strategy for type A intramural haematoma and acute aortic dissection with thrombosed false lumen of the ascending aorta: a Japanese single-centre experience. Eur J Cardiothorac Surg 2020;58:590-7.

30. Sadamatsu K, Takase S, Sagara S, et al. Initial medical management in acute type A aortic dissection patients with a thrombosed false lumen in the ascending aorta combining intramural hematoma and retrograde dissection from the descending to the ascending aorta. Eur Heart J Acute Cardiovasc Care 2020;9:S13-20.

31. Desai ND, Gottret JP, Szeto WY, et al. Impact of timing on major complications after thoracic endovascular aortic repair for acute type B aortic dissection. J Thorac Cardiovasc Surg 2015;149:S151-6.

32. Kuo EC, Veranyan N, Johnson CE, et al. Impact of proximal seal zone length and intramural hematoma on clinical outcomes and aortic remodeling after thoracic endovascular aortic repair for aortic dissections. J Vasc Surg 2019;69:987-95.

Cite this article as: $\mathrm{Li}$ J, Zhang $\mathrm{X}$, Peng $\mathrm{Y}$, Wang L, Wang T, Li X, He H, Li Q, Shu C. Endovascular repair for retrograde type A intramural hematoma with focal intimal disruption in descending aorta. J Thorac Dis 2021;13(7):4250-4259. doi: 10.21037/jtd-21-574 